version 22.7.2013

\title{
Solution of the relativistic bound state problem for hadrons
}

\author{
H.P. Morsch 1
}

National Centre for Nuclear Research, Pl-00681 Warsaw, Poland

\begin{abstract}
A second order extension of the QED Lagrangian (including boson-boson coupling) has been used to describe $q \bar{q}$ hadrons. Assuming massless elementary fermions (quantons) this results in a finite theory without open parameters, which may be regarded as a fundamental description of the strong interaction. Two potentials are deduced, a boson-exchange potential and one, which can be identified with the known confinement potential in hadrons. This formalism has been applied the mesonic systems $\omega(782), \Phi(1020), J / \psi(3097)$ and $\Upsilon(9460)$, for which a good description is obtained.

The most important results are: 1 . The confinement of hadrons is not due to colour, but is a general property of relativistic bound states. 2. Massive quarks in the Standard Model (QCD) are understood as effective fermions with a mass given by the binding energy in the boson-exchange potential.

PACS/ keywords: 11.15.-q, 12.40.-y, 14.40.Cs/ Bound state description of hadrons based on a second order Lagrangian with massless fermions (quantons) and twoboson coupling. Confinement and boson-exchange potential. Quarks understood as effective fermions with masses given by bound state energies. Mesonic systems $\omega(782), \Phi(1020), J / \Psi(3097)$ and $\Upsilon(9460)$ studied.
\end{abstract}

To study fundamental forces, nature provides us with hadrons and leptons, which form the constituents of matter, but also with composite systems, nuclei, atoms, and gravitational states in form of solar and galactic systems. For the description of these stable and massive systems as bound states a relativistic theory is needed, since the elementary constituents of these states are relativistic. However, relativistic bound state problems are generally difficult to solve, see e.g. Salpeter and Bethe [1], and could not be tackled so far for particle bound states (see the discussion in ref. [2]).

\footnotetext{
${ }^{1}$ permanent address: HOFF, Brockmüllerstr. 11, D-52428 Jülich, Germany

E-mail: h.p.morsch@gmx.de
} 
Instead, powerful effective theories have been developed, which are contained (except gravitation) in the Standard Model of particle physics [3] (SM). In these divergent first order field theories particle bound states are included effectively by a number of parameters including nine masses of 'elementary' particles. Only in QED bound states have been calculated from the Coulomb potential. But also in this theory the magnitude of the coupling constant $\alpha$ is not understood from first principles.

To understand the underlying mechanisms as well as the parameters needed in first order theories, a more fundamental theory should exist, in which these features are explained. If we demand further a real physical understanding of the development of particle systems in the universe, this theory should most likely be finite, since nature develops in a smooth way without singularities. Such a theory is expected to be based on a Lagrangian including higher order fields. This can be seen for example from the mass of light hadrons, which is much larger than the underlying quark masses.

However, in spite of an evident need for higher order theories, there is a strong belief that Lagrangians describing fundamental forces can be only first order. This is correct for divergent field theories, since the inclusion of higher order terms destroys the renormalisability of the theory. But this argument is not valid, if a finite theory is constructed. Another argument against the use of higher order theories is that in such theories Lagrangians with higher field derivatives [4] are required, which can lead to unphysical solutions (ghosts). But it should be realised that the problem is not the use of higher order theories in principle, but to find a form of the Lagrangian, in which all important criteria of relativistic theories are respected, like gauge invariance and energy-momentum conservation.

Recently, a second order theory has been developed by extending the QED Lagrangian by boson-boson coupling [5]. This formalism fulfills the above criteria of a relativistic theory and can be regarded as a fundamental description of the electric force in light atoms. However, for hadronic bound states the requirements are still higher and ask also for massless elementary fermions (quantons). Then, the mass of $q \bar{q}$ bound states has to be entirely due to binding energy.

An interesting question is, whether in a fundamental theory of hadrons the colour degree of freedom is really needed (since hadrons are colour neutral). The answer depends on 
confinement. If it would be due to colour (as often assumed [6]), a non-Abelian theory with colour would be needed. However, already in the description of atomic systems a confinement potential has been found [5], suggesting that confinement is a basic property of bound states of relativistic particles.

The Lagrangian may be written in the form

$$
\mathcal{L}=\frac{1}{\tilde{m}^{2}} \bar{\Psi} i \gamma_{\mu} D^{\mu} D_{\nu} D^{\nu} \Psi-\frac{1}{4} F_{\mu \nu} F^{\mu \nu}
$$

where $\tilde{m}$ is a mass parameter and $\Psi$ in general a two-component fermion field $\Psi=\left(\Psi^{+}\right.$ $\left.\Psi^{o}\right)$ and $\bar{\Psi}=\left(\Psi^{-} \bar{\Psi}^{o}\right)$ with charged and neutral part. Vector boson fields $A_{\mu}$ with charge coupling $\mathrm{g}$ are contained in the covariant derivatives $D_{\mu}=\partial_{\mu}-i g A_{\mu}$ and the Abelian field strength tensor $F^{\mu \nu}=\partial^{\mu} A^{\nu}-\partial^{\nu} A^{\mu}$.

We insert $D^{\mu}=\partial^{\mu}-i g A^{\mu}$ and $D_{\nu} D^{\nu}=\partial_{\nu} \partial^{\nu}-i g\left(A_{\nu} \partial^{\nu}+\partial_{\nu} A^{\nu}\right)-g^{2} A_{\nu} A^{\nu}$ in eq. (1) and obtain for the first term of $\mathcal{L}$

$$
\begin{gathered}
\mathcal{L}_{1}=\frac{1}{\tilde{m}^{2}} \bar{\Psi} i \gamma_{\mu} D^{\mu} D_{\nu} D^{\nu} \Psi=\frac{i}{\tilde{m}^{2}} \bar{\Psi} \gamma_{\mu} \partial^{\mu} \partial_{\nu} \partial^{\nu} \Psi+\frac{g}{\tilde{m}^{2}} \bar{\Psi} \gamma_{\mu} A^{\mu} \partial_{\nu} \partial^{\nu} \Psi \\
+\frac{g}{\tilde{m}^{2}} \bar{\Psi} \gamma_{\mu} \partial^{\mu} A_{\nu} \partial^{\nu} \Psi+\frac{g}{\tilde{m}^{2}} \bar{\Psi} \gamma_{\mu} \partial^{\mu} \partial_{\nu} A^{\nu} \Psi-\frac{i g^{2}}{\tilde{m}^{2}} \bar{\Psi} \gamma_{\mu} A^{\mu} A_{\nu} \partial^{\nu} \Psi \\
-\frac{i g^{2}}{\tilde{m}^{2}} \bar{\Psi} \gamma_{\mu} A^{\mu} \partial_{\nu} A^{\nu} \Psi-\frac{i g^{2}}{\tilde{m}^{2}} \bar{\Psi} \gamma_{\mu} \partial^{\mu} A_{\nu} A^{\nu} \Psi-\frac{g^{3}}{\tilde{m}^{2}} \bar{\Psi} \gamma_{\mu} A^{\mu} A_{\nu} A^{\nu} \Psi
\end{gathered}
$$

The gauge condition $\partial_{\mu} A^{\mu}=0$ used for simpler Lagrangians (as in QED) is replaced in our case by $\partial\left(\partial_{\nu} A^{\nu}\right)=0$.

In eq. (2) the number of field derivatives and boson couplings varies between the first and last term. This shows that the various terms are related to different kinetic situations, pointing to a rather complex dynamics of the system.

Contributions to stationary solutions have been studied by using the standard method of evaluating fermion matrix elements (or ground state expectation values) of field operators [7] derived from generalised Feynman diagrams. These can be written in the form $\mathcal{M}\left(p^{\prime}-p\right)=<$ g.s.| $K(q) \mid$ g.s. $>\sim \bar{\psi}\left(p^{\prime}\right) K(q) \psi(p)$, where $\psi(p)$ is a fermionic wave function $\psi(p)=\frac{1}{\tilde{m}^{3 / 2}} \Psi\left(p_{1}\right) \Psi\left(p_{2}\right)$ and $K\left(q=p^{\prime}-p\right)$ a kernel, which is expressed by $K(q)=\frac{1}{\tilde{m}^{2(n+1)}}\left[O^{n}(q) O^{n}(q)\right]$, where $\mathrm{n}$ is the number of boson fields and derivatives in eq. (2) (in the present case $n=3$ ). 
For the construction of stationary states we expect contributions mainly from terms of the Lagrangian (2), which contain static fields (without derivatives). This is the case only for the last term $\mathcal{L}_{1,8}=-\frac{1}{\tilde{m}^{2}} \bar{\Psi} g^{3} \gamma_{\mu} A^{\mu} A_{\nu} A^{\nu} \Psi$ and leads to a matrix element $\mathcal{M}_{3 g}$, which contains three boson fields on the right and left

$$
\mathcal{M}_{3 g}=\frac{-\alpha^{3}}{\tilde{m}^{8}} \bar{\psi}\left(p^{\prime}\right) \gamma_{\mu} A^{\mu}(q) A_{\nu}(q) A^{\nu}(q) A_{\sigma}(q) A^{\sigma}(q) \gamma_{\rho} A^{\rho}(q) \psi(p)
$$

where $\alpha=g^{2} / 4 \pi$. A comparable matrix element in a first order theory may be written in the form $\mathcal{M}_{\text {f.o. }}=\frac{-\alpha}{\tilde{m}^{4}} \bar{\psi}\left(p^{\prime}\right) \gamma_{\mu} A^{\mu}(q) \gamma_{\rho} A^{\rho}(q) \psi(p)$, giving rise to a (boson-exchange) interaction of vector structure $v_{v}(q) \sim \alpha A_{\mu}(q) A^{\rho}(q)$ (but only for equal times of the two boson fields, which means in the non-relativistic limit). Differently, in eq. (3) three interactions of scalar and vector structure $V_{\mu}^{\nu}(q) \sim \alpha A_{\mu}(q) A^{\nu}(q)$ are involved. In a dual picture the two boson fields, which appear twice (on the left and right side of $\mathcal{M}_{3 g}$ ) can be regarded (analoguous to the fermion wave function $\psi(p)$ ) as bosonic (quasi) wave functions 2 $W_{\mu}^{\nu}(q)=\frac{1}{\tilde{m}} A_{\mu}(q) A^{\nu}(q)$. The fact that boson fields can be combined to wave functions leads quite naturally to a finite theory, in which the wave functions are normalised.

The physical picture of $\mathcal{M}_{3 g}$ is that for the lowest energy state of a relativistic bound state system the fermions interact only inside the two-boson density (which leads to a boundary condition discussed below) and feel therefore three interactions. A single boson-exchange interaction is possible only in dynamical situations, see the terms 2-4 in eq. (2), which do not lead to a bound state potential.

The $\gamma$-matrices can be removed (contracted) by adding a matrix element with interchanged $\mu$ and $\rho$ (according to $\left.\frac{1}{2}\left(\gamma_{\mu} \gamma_{\rho}+\gamma_{\rho} \gamma_{\mu}\right)=g_{\mu \rho}\right)$. Further, an equal time requirement of the two-boson fields (to reach overlap) allows to replace all fermion four-vectors 3 by three-vectors in momentum or r-space. Correspondingly, the boson wave functions $W_{\mu}^{\nu}(q)$ and the remaining (boson-exchange) interaction $V_{\mu}^{\nu}(q)$ are reduced to $w_{s, v}(q)$ and $v_{v}(q) \sim w_{v}(q)$, which are two-dimensional. This yields

$$
\mathcal{M}_{3 g}=\frac{-\alpha^{3}}{\tilde{m}^{5}} \bar{\psi}\left(p^{\prime}\right) w_{s, v}(q) v_{v}(q) w_{s, v}(q) \psi(p) .
$$

Writing the matrix element by $\mathcal{M}_{3 g}=\bar{\psi}\left(p^{\prime}\right) V_{3 g}(q) \psi(p)$ we obtain a three-boson potential

$$
V_{3 g}^{s, v}(q)=\frac{-\alpha^{3}}{\tilde{m}^{2}} w_{s, v}^{2}(q) v_{v}(q)
$$

\footnotetext{
${ }^{2}$ leading to boson (quasi) densities $w^{2}(q)$ with dimension $[\mathrm{GeV}]^{2}$.

${ }^{3}$ in a $(t, \vec{r})$ representation
} 
Fourier transformation to r-space leads to a folding potential

$$
V_{3 g}^{s, v}(r)=-\frac{\alpha^{3} \hbar}{\tilde{m}} \int d r^{\prime} w_{s, v}^{2}\left(r^{\prime}\right) v_{v}\left(r-r^{\prime}\right)
$$

Such a form has been used to describe elastic and inelastic hadron processes [8].

The bosonic part of eq. (4) can also be written in the form of a matrix element, in which the wave functions $w_{s, v}(q)$ are connected by $v_{v}(q)$

$$
\mathcal{M}^{g}=\frac{-\alpha^{3}}{\tilde{m}^{2}} w_{s, v}(q) v_{v}(q) w_{s, v}(q)
$$

This matrix element shows binding of two bosons in the potential $v_{v}(q)$, consequently $\partial^{2} w_{s}(q)$ is related to their kinetic energy. The contribution from the vector part $\partial^{2} w_{v}(q)$ cancels out as a consequence of the gauge condition. Below it will be shown that this implies also the existence of a static two-boson potential $V_{2 g}(q)$.

From the general structure of the fermion matrix element in eq. (4) one can see that there are two fundamental s-states (with quantum numbers $\left(J^{\pi}=1^{-}\right)$) with scalar and vector boson wave functions $w_{s, v}(r)$ and corresponding fermion wave functions $4 \psi_{s, v}(r) \sim w_{s, v}(r)$ normalised by $4 \pi \int r^{2} d r \psi_{s, v}(r)=1$. Further, there are $0^{+}$states with p-wave functions $\psi_{L=1}(r)$, which are not considered in the present paper.

Orthogonality of the total wave functions requires that the boson wave functions $w_{s}(r)$ and $w_{v}(r)$ are orthogonal and lead to a vanishing radial matrix element

$$
<r_{w_{s}, w_{v}}^{2}>=\int r^{3} d r w_{s}(r) w_{v}(r)=0
$$

To satisfy this condition, $w_{v}(r)$ may be written in the form of a $\mathrm{p}$-wave function

$$
w_{v}(r)=w_{v, o}\left[w_{s}(r)+\beta R \frac{d w_{s}(r)}{d r}\right],
$$

where $w_{v, o}$ is obtained from the normalisation $2 \pi \int r d r w_{v}^{2}(r)=1$ and $\beta R$ from eq. (8).

To evaluate the potentials $V_{3 g}^{s, v}(r)$, the boson wave functions $w_{s, v}(r)$ have to be determined. To achieve this, a boundary condition can be formulated by requiring that the interaction takes place inside the volume of the strongest bound state. As a consequence, the corresponding boson-exchange potential (6) should be proportional to the density $\psi^{2}(r) \sim w_{s}^{2}(r)$, leading to

$$
c w_{s}^{2}(r) \sim\left|V_{3 g}^{v}(r)\right| .
$$

\footnotetext{
${ }^{4}$ for the radial wave functions $\bar{\psi}(r)=\psi(r)$.
} 
Both constraints (8) and (10) can be satisfied by a boson wave function of the form

$$
w_{s}(r)=w_{s_{o}} \exp \left\{-(r / b)^{\kappa}\right\}
$$

where $w_{s_{o}}$ is the normalisation factor $1 /\left(4 \pi \int r d r w_{s}^{2}(r)\right)$. The boson-exchange interaction $v_{v}(r)$ is given by $v_{v}(r)=\hbar w_{v}(r)$.

To generate a stable bound state, the potential $V_{3 g}^{s, v}(r)$ is not sufficient to keep the bosons confined. The other terms of the Lagrangian (2) show kinematic situations, in which bosons and/or fermions are in motion. Nevertheless, term 6 may be written in the form $\mathcal{L}_{1,6}=-\frac{i g^{2}}{\bar{m}^{2}} \bar{\Psi} \gamma_{\mu} A^{\mu}\left(\partial_{\nu} A^{\nu}\right) \Psi-\frac{i g^{2}}{\bar{m}^{2}} \bar{\Psi} \gamma_{\mu} A^{\mu} A_{\nu} \partial^{\nu} \Psi$ and gives rise to another bound state potential.

The first term of $\mathcal{L}_{1,6}$ leads to

$$
\mathcal{M}_{2 g}=\frac{\alpha^{2}}{\tilde{m}^{8}} \bar{\psi}\left(p^{\prime}\right) \gamma_{\mu} A^{\mu}(q)\left(\partial_{\nu} A^{\nu}(q)\right) \gamma_{\rho} A^{\rho}(q)\left(\partial_{\sigma} A^{\sigma}(q)\right) \psi(p) .
$$

Using the gauge condition we can write $\left(\partial_{\nu} A^{\nu}(q)\right)\left(\partial_{\sigma} A^{\sigma}(q)\right)=\frac{1}{2} \partial_{\nu}\left[\partial_{\sigma}\left(A_{\mu} A^{\mu}\right)^{\sigma}\right]^{\nu}$. After contracting the $\gamma$-matrices and reducing the fermion and boson vectors by one dimension as discussed for $\mathcal{M}_{3 g}$, this yields

$$
\mathcal{M}_{2 g}=\frac{\alpha^{2}}{2 \tilde{m}^{6}} \bar{\psi}\left(p^{\prime}\right) w_{s}(q) \partial^{2} w_{s}(q) \psi(p) .
$$

Since the two bosons are bound, see eq. (7), $\partial^{2} w_{s}(q) / 2 \tilde{m}$ is related to their kinetic energy distribution. According to the virial theorem this implies also the existence of a static two-boson potential $V_{2 g}(q)$.

In a transformation to r-space the bosonic part of eq. (13) gives rise to a Hamiltonian of the form

$$
-\frac{\alpha^{2} \tilde{m}<r_{w_{s}}^{2}>F_{2 g}}{4}\left(\frac{d^{2} w_{s}(r)}{d r^{2}}+\frac{2}{r} \frac{d w_{s}(r)}{d r}\right)+V_{2 g}(r) w_{s}(r)=E_{i} w_{s}(r),
$$

where the factor $F_{2 g}$ is due to the Fourier transformation of the boson kinetic energy, $<r_{w_{s}}^{2}>$ the radius square of the boson density and $w_{s}(r)$ the Fourier transform of $w_{s}(q)$. The potential $V_{2 g}(r)$ is given by

$$
V_{2 g}(r)=\frac{\alpha^{2} \tilde{m}<r_{w_{s}}^{2}>F_{2 g}}{4}\left(\frac{d^{2} w_{s}(r)}{d r^{2}}+\frac{2}{r} \frac{d w_{s}(r)}{d r}\right) \frac{1}{w_{s}(r)}+E_{o},
$$


where $E_{o}=0$ is used to make a connection to the vacuum (state without binding between the quantons and therefore $\left.E_{v a c}=0\right)$. A similar potential involving $w_{v}(q)$ deduced from $\mathcal{L}_{1,7}$ yields negligible contribution to the binding energy. All other terms of the Lagrangian (2) do not contribute to bound state potentials.

The implications of using massless fermions are very strong and can be summarized as follows: First, the vacuum of the theory is the absolute vacuum with average energy $E_{v a c}=0$. This is consistent with the low energy density of the universe deduced from astrophysical observations. Second, the lowest energy solution in $V_{2 g}(r)$ is the vacuum and therefore $E_{o}=E_{v a c}=0$. By this condition the absolute height of $V_{2 g}(r)$ is fixed. Third, by rewriting eq. (13) in the form $\mathcal{M}_{2 g}=\frac{\alpha^{2}}{2 \tilde{m}^{6}} w_{s}(q)\left\{\bar{\psi}\left(p^{\prime}\right) \psi(p)\right\} \partial^{2} w_{s}(q)$, one can see that fermion-antifermion pairs can be created during the dynamical overlap of two fluctuating boson fields. By this mechanism stable particles can be created out of the absolute vacuum. These facts are consistent with the requirement for a fundamental theory.

An important fact is that $V_{2 g}(r)$ can be identified with the confinement potential in hadron potential models [9]. This will be shown in a comparison with the confinement potential from lattice QCD simulations [10] and the discussion of quark masses.

$V_{2 g}(r)$ can also be written in a different form

$$
V_{2 g}(r)=\frac{\alpha^{2} \hbar^{2} F_{2 g}}{4 \tilde{m}}\left(\frac{d^{2} w_{s}(r)}{d r^{2}}+\frac{2}{r} \frac{d w_{s}(r)}{d r}\right) \frac{1}{w_{s}(r)}+E_{o} .
$$

This leads to the condition

$$
R a t=\frac{\hbar^{2}}{\tilde{m}^{2}<r_{w_{s}}^{2}>}=1 .
$$

A last constraint is related to energy-momentum conservation in relativistic systems, indicating that for binding in $V_{3 g}^{s}(r)$ the total energy of the system is not increased, the negative fermion and boson binding energies $E_{f}^{s}$ and $E_{g}$ have to be compensated by the root mean square momenta of the corresponding potentials

$$
<q_{V_{3 g}}^{2}>^{1 / 2}+<q_{v_{v}}^{2}>^{1 / 2}=-\left(E_{f}^{s}+E_{g}\right)
$$

However, for the confinement potential $V_{2 g}(r)$ this condition is not valid. Therefore, the constraint (18) can be applied only for the binding potentials $V(q)=V_{3 g}(q)$ and $v_{v}(q)$ with $\left\langle q_{V}^{2}>=f_{\text {red }} \int d q q^{3} V(q) / \int d q q V(q)\right.$ and $f_{\text {red }}=E_{f}^{s} /\left(E_{f}^{s}+E_{g}\right)$. 
The fermion mass of the system is defined by the energy to balance binding

$$
M_{n}^{s, v}=-E_{f_{s, v}}^{3 g}+E_{f_{n}}^{2 g},
$$

where $E_{f_{s, v}}^{3 g}$ is the negative binding energy in $V_{3 g}^{s, v}(r)$ (for these potentials only the lowest state is discussed here) and $E_{f_{n}}^{2 g}$ are positive binding energies for different (excited) states in $V_{2 g}(r)$. This shows two types of mass generation, binding in the Coulomb like potential $V_{3 g}(r)$ and dynamical mass generation in $V_{2 g}(r)$.

In the whole formalism there are finally four constraints (8), (10), (17) and (18), by which all open parameters, shape parameter $\kappa$, slope (or size) parameter $b$ and the coupling constant $\alpha$, are determined within rather small ambiguities. In addition, the different flavour states in the quark mode 5 can be related by a vacuum sum rule similar to that applied in ref. [5], which indicates that in principle a complete solution of the relativistic bound state problem for all states is achieved. Below it will be shown that the need for massless elementary fermions in the present formalism is entirely consistent with the requirement of finite quark masses in the SM.

The above formalism has been applied to $q \bar{q}$ mesons (of different flavour structure in the quark model) $\omega(782), \Phi(1020)$, charmonium $J / \Psi(3097)$ and bottonium $\Upsilon(9460)$ including excited states. The potentials $V_{3 g}(r)$ and $V_{2 g}(r)$ have been determined by adjusting the open parameters to fulfill the constraints discussed above. Remaining uncertainties have been reduced by fine-adjustment of the factor $F_{2 g}$ in the confinement potential $V_{2 g}(r)$ to fit the spectrum of radial excitations.

Results on the radial dependence of densities and potentials are given in fig. 1 for the $\omega(782)$ system. In the upper part the interaction $v_{v}(r)$ is given by the solid line. Compared to the Coulomb potential $v_{\text {coul }}(r)=\hbar / r$ (dot-dashed line) there are no divergencies for $r \rightarrow 0$ and $\infty$, in agreement with the demand of a finite theory.

In the middle part a comparison of the density $w_{s}^{2}(r)$ (dot-dashed line) with the potentials $V_{3 g}^{s}(r)$ (dashed line) and $V_{3 g}^{v}(r)$ (solid line) is made. We see that condition (10) for the vector potential is reasonably well fulfilled at larger radii. This indicates that the

\footnotetext{
${ }^{5}$ the notion of flavour from the quark model is kept in the present approach to characterise systems of different slope parameter $b$.
} 
Table 1: Results for mesonic systems, $\omega(782), \Phi(1020), J / \psi(3097)$, and $\Upsilon(9460)$ including excited states, in comparison with the data [3]. Masses and binding energies are given in $\mathrm{GeV}, b$ in $\mathrm{fm}$, and the mean radius squares in $\mathrm{fm}^{2} . \alpha_{e q}$ is the equivalent coupling constant in the Coulomb potential.

\begin{tabular}{|c|c|c|c|c|c|c|c|c|}
\hline System & $M_{1}^{s}$ & $M_{2}^{s}$ & $M_{3}^{s}$ & $M_{4}^{s}$ & $M_{1}^{v}$ & $M_{1}^{\exp }$ & $M_{2}^{e x p}$ & $M_{3}^{e x p}$ \\
\hline$\omega$ & 0.78 & 1.42 & 1.93 & & 1.3 & 0.782 & $1.42 \pm 0.03$ & \\
\hline$\Phi$ & 1.02 & 1.68 & 2.20 & & 2.0 & 1.02 & $1.68 \pm 0.02$ & \\
\hline$J / \psi$ & 3.10 & 3.69 & 4.16 & 4.58 & 8.1 & 3.097 & 3.686 & $4.16 \pm 0.02$ \\
\hline$\Upsilon$ & 9.46 & 10.02 & 10.46 & 10.8 & 26.6 & 9.46 & 10.023 & 10.355 \\
\hline System & $\kappa$ & $b$ & $\alpha$ & $\alpha_{e q}^{*}$ & $E_{g}$ & $E_{3 g}^{s}$ & $m_{\text {quark }}$ & $<r_{w_{s}}^{2}>$ \\
\hline$\omega$ & 1.4 & 0.589 & 0.65 & 0.05 & -0.026 & -0.013 & 0.0065 & 0.256 \\
\hline$\Phi$ & 1.4 & 0.450 & 1.50 & 0.65 & -0.43 & -0.216 & 0.108 & 0.150 \\
\hline$J / \psi$ & 1.4 & 0.148 & 2.32 & 2.46 & -4.82 & -2.42 & 1.21 & 0.016 \\
\hline$\Upsilon$ & 1.4 & 0.049 & 2.49 & 3.04 & -18.4 & -9.0 & 4.5 & 0.0017 \\
\hline
\end{tabular}

bosonic wave function $w_{s}(r)$ is well described by the radial form in eq. (111) and that also relation (9) between $w_{s}(r)$ and $w_{v}(r)$ is correct.

In the lower part of fig. 1 the deduced confinement potential $V_{2 g}(r)$ is shown. It is characterized by a close to linear form at larger radii, as expected from ref. [9, 10]. Resulting masses and parameters for different systems are given in table 1. Although the binding energies are quite different, in all cases a satisfactory agreement of the various quantities is obtained, which fulfill all boundary conditions. A similar plot as in fig. 1 is shown for the bottonium system in fig. 2. Apart from a very different radial extent of the two systems the only important difference is the relative size of the confinement potential, which is drastically reduced for the heavy system due to a very different dynamics.

A comparison of the deduced confinement potential $V_{2 g}(r)$ with the lattice QCD simulations of Bali et al. [10] (solid points with error bars) is shown in fig. 2. This potential has the same form as the confinement potential $V_{\text {conf }}(r) \sim-\alpha / r+l \cdot r$ deduced from potential models [9]. The fact that very similar results are deduced from theories with and without colour indicates clearly that the confinement of hadrons is not due to colour (as assumed 
in ref. [6] but without clear understanding of the mechanisms involved), but represents a general property of relativistic bound states.

The question of a vector or scalar structure of the confinement potential can be studied by looking at the splitting of p-wave states in charmonium and bottonium, see ref. [11]. From the existing data neither a vector nor a scalar structure is found, supporting strongly a derivative structure of the potential $V_{2 g}(r)$, as found in the present approach.

An important point is the need for finite quark masses in the SM (QCD), which should be understood in the present more fundamental approach. These masses have been estimated in different models, as e.g. in QCD inspired potential models [9] (more details can be found in ref. [3]). The empirical form $V_{\text {conf }}(r) \sim-\alpha / r+l \cdot r$ assumed in these models is consistent with $V_{2 g}(r)$; therefore, the quark masses have to be related to the binding energy in $V_{3 g}^{s}(r)$. This leads to the relation 6

$$
m_{\text {quark }}=-\frac{1}{2} E_{3 g}^{s}
$$

The resulting quark masses are given in table 1 and are compared with the extracted masses [3] in fig. 3. An excellent agreement is obtained. This is clear indication that the need for massive fermions (quarks) in the first order theory (QCD) is perfectly consistent with the assumption of massless elementary fermions in the present approach. Thus, the quarks can be understood as effective fermions with masses related to the binding energy in the bound state potential $V_{3 g}^{s}(r)$.

Concerning an interpretation of the quark masses as due to the Higgs-mechanism [12], such an explanation (which demands an extra high energetic background field) is not needed. Further, the flavour structure of mesons comes out naturally in the present approach. Therefore, supersymmetric extensions of the SM, which predict a new regime of super-symmetric particles at high energies, are also not needed. This confirms the general view that a fundamental theory must have a very simple structure.

\footnotetext{
${ }^{6}$ this expression is independent of using an Abelian or non-Abelian structure of the Lagrangian. In an Abelian theory massive "quarks" have the same charge as the quantons in eq. (11).
} 
In conclusion, although the SM yields an excellent description of many particle properties, it is an effective theory with parameters (quark masses), which are not well understood from first principles. To get a correct understanding of the nature of these parameters, a more fundamental theory of hadrons is required. This has been achieved in the present approach, in which the colour degree of freedom as well as Higgs and supersymmetric fields are not needed. Preliminary results from an application to different fundamental forces can be found in ref. [14].

The author is very grateful to many colleagues for fruitful discussions, valuable comments and the help in formal derivations. Special thanks to P. Decowski and P. Zupranski for numerous conversations and encouragements and B. Loiseau for his help with the formulation of the Lagrangian and matrix elements. 


\section{References}

[1] E.E. Salpeter and H.A. Bethe, Phys. Rev. 84, 1232 (1951)

[2] St.G. Glazek, Acta Phys. Polonica B 24, 1315 (1993); and refs. therein

[3] Review of particle properties, K. Nakamura et al., J. Phys. G 37, 075021 (2010); http://pdg.lbl.gov/ and refs. therein

[4] J.Z. Simon, Phys. Rev. D 41, 3720 (1990); A. Foussats, E. Manavella, C. Repetto, O.P. Zandron, and O.S. Zandron, Int. J. theor. Phys. 34, 1 (1995); V.V. Nesterenko, J. Phys. A: Math. Gen. 22, 1673 (1989); and refs. therein

[5] H.P. Morsch, arXiv 1104.2574 [hep-ph], see new replacement

[6] see eg.g. the discussion in J. Greensite, Prog. Part. Nucl. Phys. 51 (2003) 1 and arXiv: hep-lat/0301023

[7] M.E. Peskin and D.V. Schroeder, "An introduction to quantum field theory", Addison-Wesley Publ. Comp. (1995)

[8] H.P. Morsch, W. Spang, and P. Decowski, Phys. Rev. C 67, 064001 (2003)

[9] R. Barbieri, R. Kögerler, Z. Kunszt, and R. Gatto, Nucl. Phys. B 105, 125 (1976); E. Eichten, K.Gottfried, T. Kinoshita, K.D. Lane, and T.M. Yan, Phys. Rev. D 17, 3090 (1978); S. Godfrey and N. Isgur, Phys. Rev. D 32, 189 (1985); D. Ebert, R.N. Faustov, and V.O. Galkin, Phys. Rev. D 67, 014027 (2003); and refs. therein

[10] G.S. Bali, K. Schilling, and A. Wachter, Phys. Rev. D 56, 2566 (1997);

G.S. Bali, B. Bolder, N. Eicker, T. Lippert, B. Orth, K. Schilling, and T. Struckmann, Phys. Rev. D 62, 054503 (2000)

[11] H.J. Schnitzer, Phys. Rev. Lett. 35, 1540 (1975)

[12] See e.g. P. Higgs, Phys. Rev. Lett. 19, 508 (1964). Note that in this formalism the divergencies of the SM are not removed.

[13] H.P. Morsch and P. Zupranski, Phys. Rev. C 61, 024002 (1999)

[14] H.P. Morsch, EPJ Web of Conferences 28, 12068 (2012), Hadron Collider Physics Symposium, Paris 2011 (open access) 

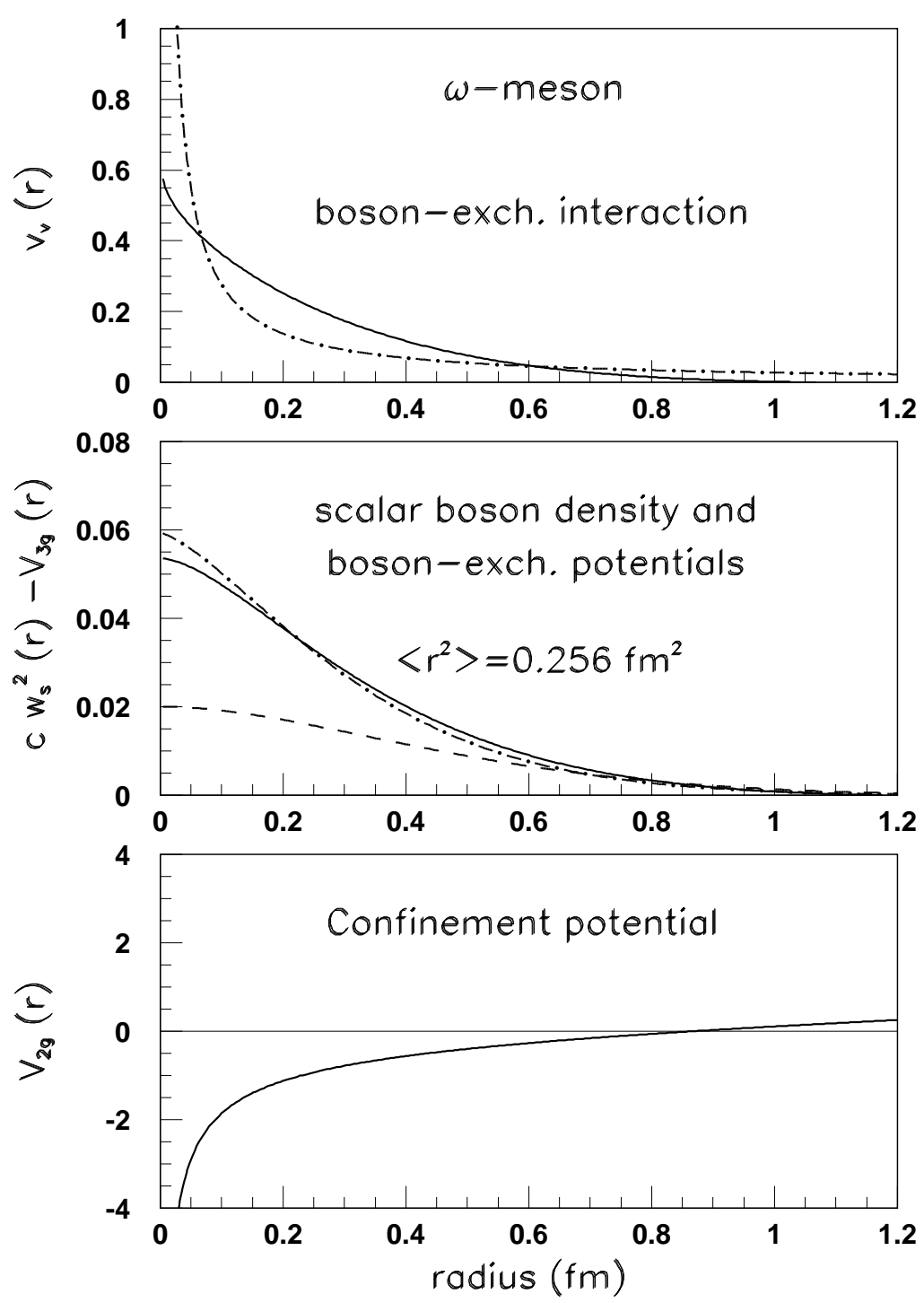

Figure 1: Self-consistent solution for the $\omega(782)$ meson system. Upper part: Interaction $w_{v}(r)$ in comparison with the Coulomb potential, given by solid and dot-dashed lines, respectively. Middle part: Bosonic density $w_{s}^{2}(r)$ and potential $\left|V_{3 g}^{v}(r)\right|$ given by the overlapping dot-dashed and solid lines, respectively, matched by the condition (10); $\left|V_{3 g}^{s}(r)\right|$ is shown by dashed line. Lower part: Deduced confinement potentials $V_{2 g}(r)$. 

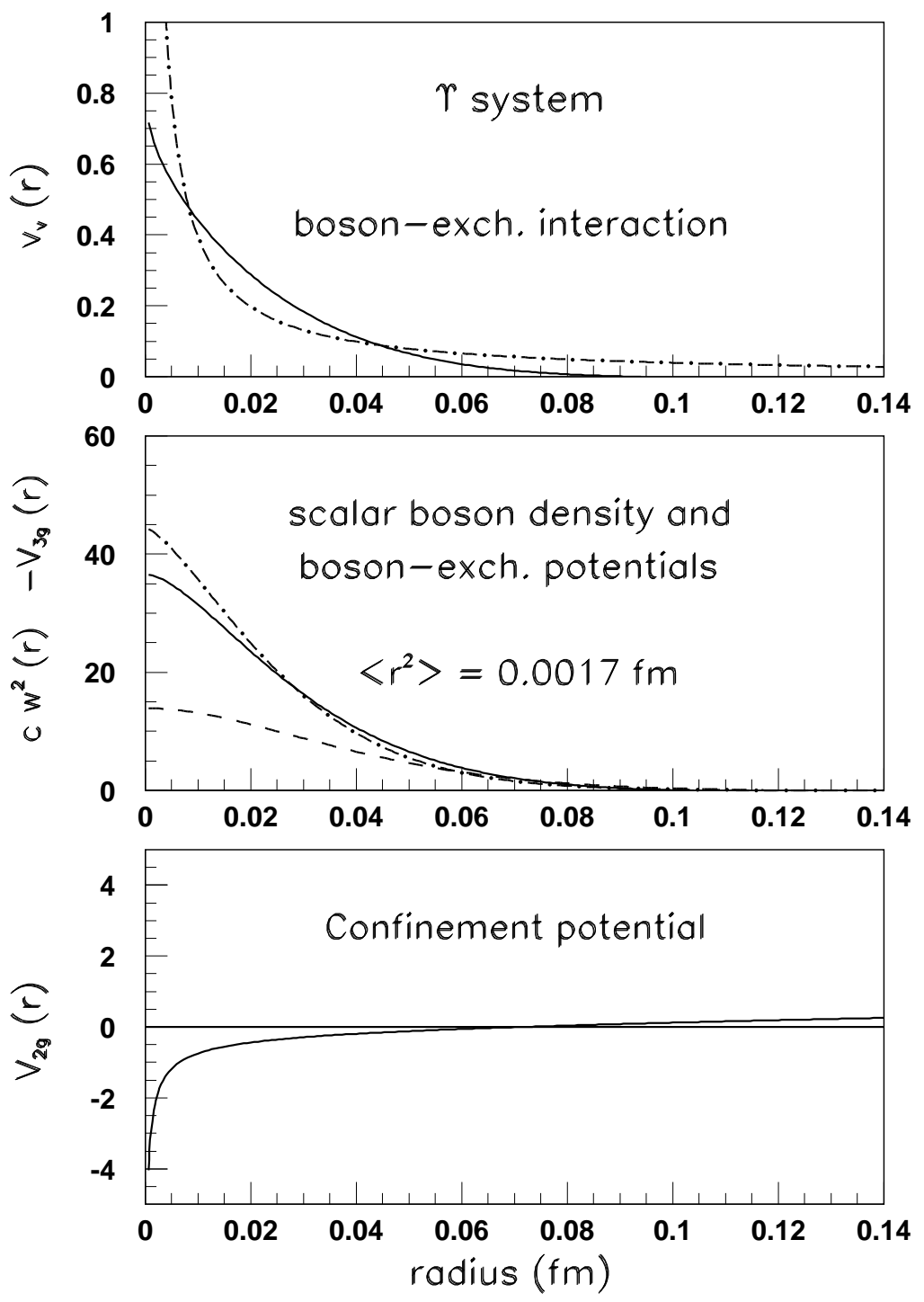

Figure 2: Same as fig. 1 for the bottonium system $\Upsilon(9460)$. 


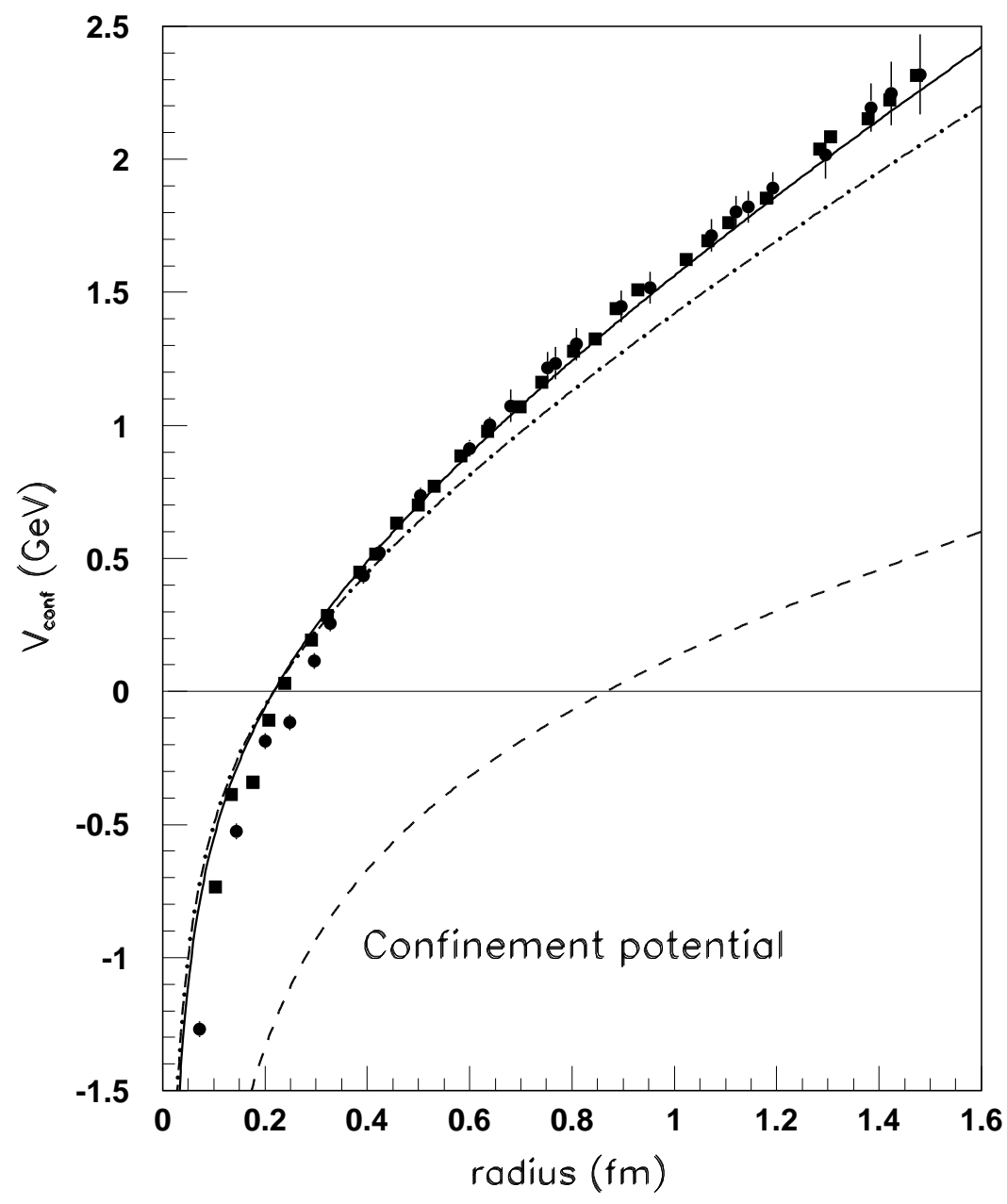

Figure 3: Comparison of the confinement potential with lattice QCD calculations. $V_{2 g}(r)$ calculated for the two mesonic systems $\omega(782)$ and charmonium $J / \psi$, given by dashed and dot-dashed line, respectively. The latter, multiplied with a factor 1.1 (solid line) shows an excellent agreement with lattice gauge simulations [10] (solid points). 


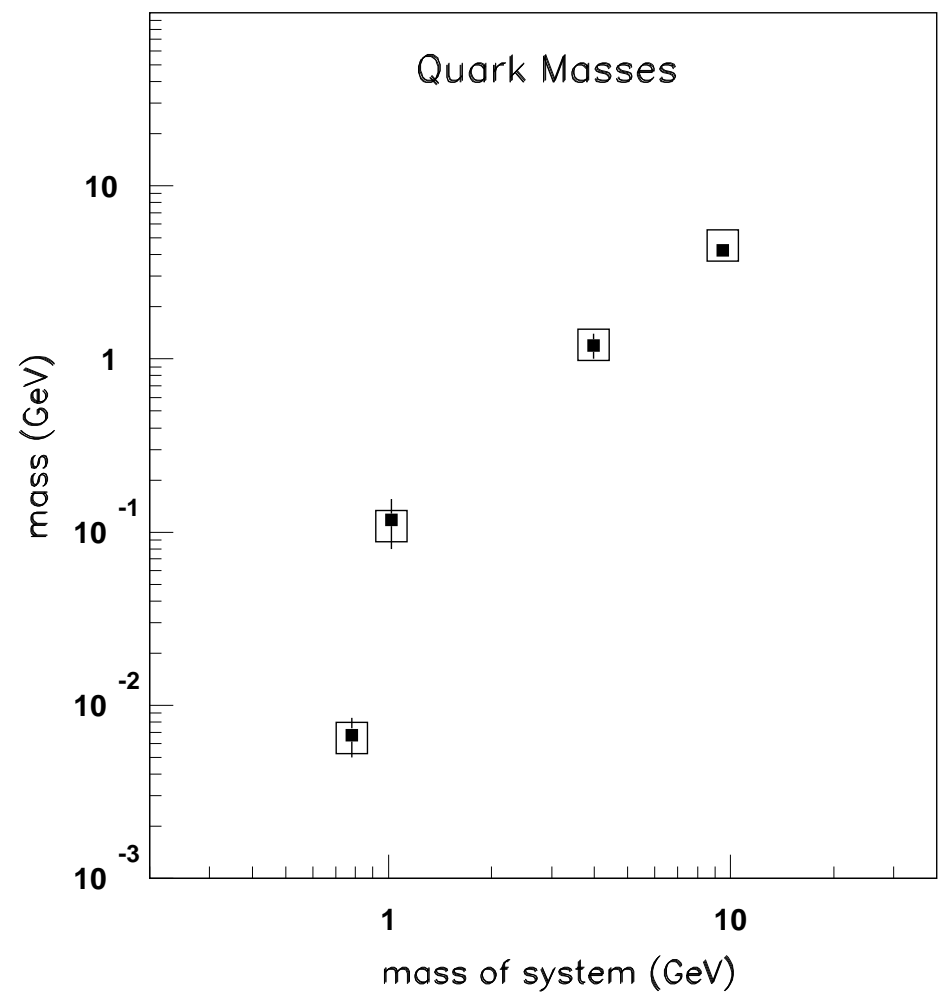

Figure 4: Quark masses as a function of the g.s. masses. The open squares show the present results using eq. (20), the solid squares with error bars give the extracted values from other sources $[3]$. 